\title{
Thermal evolution of the band edges of 6H-SiC: X-ray methods compared to the optical band gap
}

\author{
P.S. Miedema ${ }^{1}$, M. Beye ${ }^{1}$, R. Könnecke ${ }^{1}$, G. Schiwietz ${ }^{1}$, A. Föhlisch ${ }^{1,2}$ \\ ${ }^{1}$ Institute for Methods and Instrumentation in Synchrotron Radiation Research G-ISRR, Helmholtz-Zentrum für \\ Materialien und Energie GmbH, Albert-Einstein-Strasse 15, 12489 Berlin, Germany \\ ${ }^{2}$ Fakultät für Physik und Astronomie, Universität Potsdam, Karl-Liebknecht-Strasse 24-25, 14476 Potsdam, \\ Germany
}

\begin{abstract}
The band gap of semiconductors like silicon and silicon carbide $(\mathrm{SiC})$ is the key for their device properties. In this research, the band gap of $6 \mathrm{H}-\mathrm{SiC}$ and its temperature dependence were analyzed with silicon $2 \mathrm{p} \mathrm{x}$-ray absorption spectroscopy (XAS), x-ray emission spectroscopy (XES) and resonant inelastic x-ray scattering (RIXS) allowing for a separate analysis of the conduction-band minimum (CBM) and valence-band maximum (VBM) components of the band gap. The temperature-dependent asymmetric band gap shrinking of $6 \mathrm{H}-\mathrm{SiC}$ was determined with a valence-band slope of $+2.45 \times 10^{-4} \mathrm{eV} / \mathrm{K}$ and a conduction-band slope of $-1.334 \times 10^{-4} \mathrm{eV} / \mathrm{K}$. The apparent asymmetry, e.g., that two thirds of the band-gap shrinking with increasing temperature is due to the VBM evolution in $6 \mathrm{H}-\mathrm{SiC}$, is similar to the asymmetry obtained for pure silicon before. The overall band gap temperature-dependence determined with XAS and non-resonant XES is compared to temperature-dependent optical studies. The core-excitonic binding energy appearing in the Si $2 p$ XAS is extracted as the main difference. In addition, the energy loss of the onset of the first band in RIXS yields to values similar to the optical band gap over the tested temperature range.
\end{abstract}

\section{Keywords}

RIXS, XAS, XES, semiconductors, silicon carbide

\section{Introduction}

Semiconductor materials are the heart of solid state electronics with devices including transistors, switches, diodes, photovoltaic cells and detectors [1]. Silicon is the workhorse for information technologies and photovoltaic photon harvesting, while $\mathrm{SiC}$ is a promising material for high power, high temperature and high frequency applications, because of its extreme thermal and chemical stability together with the large electron saturation velocity and mobility [3,4] and $\mathrm{SiC}$ has applications in light-emitting diodes [2-4], temperature sensors [5] and as neutron detectors [6, 7]. The most stable of about 100 different $\mathrm{SiC}$ polytypes, the hexagonal $6 \mathrm{H}-\mathrm{SiC}$ containing six $\mathrm{SiC}$ pairs per unit cell with the stacking sequence ABCACB [8] is the subject of this study.

The physical characteristics of a semiconductor are governed by the electronic band structure and the band gap is the key property. This band gap is defined as the difference in the energy between the lowest point of the conduction band (conduction band minimum, CBM) and the highest point in the valence band (valence band maximum, VBM). Silicon and $\mathrm{SiC}$ both have an indirect band gap meaning that the VBM and CBM are separated in k-space. Recently there was a theoretical study about the phonon-assisted optical absorption in silicon, which showed the difficulties in calculations of indirect band gap materials [9]. The indirect band gap is $1.12 \mathrm{eV}$ for silicon. For different polytypes of $\mathrm{SiC}$ the indirect band gap ranges from 2.3 to $3.1 \mathrm{eV}$ depending on the crystal structure of $\mathrm{SiC}[10]$ with a value of about $2.9-3.1 \mathrm{eV}$ for $6 \mathrm{H}-\mathrm{SiC}$ [11].

The temperature-dependence of the band gap is a material-specific property, which is of considerable practical interest for the operation of semiconductor devices while it is physically driven by the electron-phonon (e-ph) coupling [12]. Thermally induced changes of the band structure and explicitly in the band gap of semiconductors arise from a trivial thermal expansion effect plus a direct e-ph interaction effect [13]. It is known from a wealth 
of experimental and theoretical results (for example $[14,15]$ ) that band gap temperature dependencies can be split in two regions: usually a quadratic or cubic dependence on temperature in the cryogenic region and a strong (nearly) linear temperature dependence above the Debye temperature. Although calculations of the temperaturedependence of the gaps based on semi-empirical e-ph interaction have appeared in the literature, the band gap energies and the linear temperature dependence are not completely reliable. Typically the calculated band-gap energies are too high [16] when compared to experimentally obtained band gaps and their fitted Varshni expressions [17] of temperature relations for band gaps. For $6 \mathrm{H}-\mathrm{SiC}$, the experimental temperature dependence of the band gap is fitted with $\mathrm{E}_{\mathrm{g}}(\mathrm{T})=\mathrm{E}_{\mathrm{g}}(0)-6.5 \times 10^{-4} \mathrm{xT}^{2} /(\mathrm{T}+1200)$ [18] with $\mathrm{E}_{\mathrm{g}}$ in $\mathrm{eV}$ and $\mathrm{T}$ in Kelvin.

Experimentally, the direct band gaps are often determined with (UV-VIS) optical studies. These optical band gaps are not directly the energetic distance of the CBM and VBM under equilibrium conditions, but these are lowered by the binding energy of the created electron-hole pair, the valence exciton [1]. Some valence exciton binding energies are shown in Table 1 and all these valence exciton binding energies are in the meV regime. In most cases such excitonic effects are neglected in the optical band gap determination, because these excitonic binding energies are small in comparison to the band gap energy and are below or in the range of the error bars of the measurements of these band gaps.

Table 1. Exciton binding energies for silicon and some SiC polytypes [19].

\begin{tabular}{|c|c|}
\hline Compound & Valence-exciton binding energy (meV) \\
\hline Silicon & 14.7 \\
\hline $3 \mathrm{C}-\mathrm{SiC}$ & 13.5 \\
\hline $4 \mathrm{H}-\mathrm{SiC}$ & 20.0 \\
\hline $6 \mathrm{H}-\mathrm{SiC}$ & 78.0 \\
\hline
\end{tabular}

In the present study we apply x-ray absorption spectroscopy (XAS), non-resonant $\mathrm{x}$-ray emission spectroscopy (XES) and resonant inelastic x-ray scattering (RIXS) spectroscopy for analysis of the CBM and VBM components of the band gap of $6 \mathrm{H}-\mathrm{SiC}$ as a function of temperature. In addition we discuss the difference between this combination of spectroscopies and optical spectroscopies in the band-gap analysis. Crystalline silicon was analyzed in a similar study, where an asymmetric band gap closing of crystalline silicon was experimentally determined with the slope of $-1.61 \times 10^{-4} \mathrm{eV} / \mathrm{K}$ for the conduction-band and $+2.93 \times 10^{-4} \mathrm{eV} / \mathrm{K}$ for the valence-band [20] and we compare the analysis of $6 \mathrm{H}-\mathrm{SiC}$ presented below with the data obtained for silicon.

\section{Experimental methods}

The back of a $6 \mathrm{H}-\mathrm{SiC}$ single crystal as obtained from MaTecK was mounted on a molybdenum plate to have good thermal contact. This molybdenum plate was heated either radiatively through a hot filament or with electron bombardment. The temperature of the sample was measured by a pyrometer on the front side of the $6 \mathrm{H}-$ $\mathrm{SiC}$ crystal, which is the side where the interaction with the $\mathrm{x}$-rays occurs.

This sample was measured with soft $\mathrm{x}$-ray non-resonant $\mathrm{x}$-ray emission spectroscopy (XES) and resonant inelastic X-ray scattering (RIXS) using our using our RIXS setup [21] at beamline UE112-PGM1 of the Bessy II synchrotron in Berlin, Germany. The photon-energy bandwidth of the beamline was $0.1 \mathrm{eV}$ and the spectrometer had a resolution of $0.2 \mathrm{eV}$ based on the measured HWHM of the elastic peak. Si $2 \mathrm{p}$ x-ray absorption spectra (XAS) and RIXS spectra using an incident x-ray energy at the Si 2p edge (101.35 eV, RIXS) and XES spectra using an incident energy above the Si $2 p$ edge of $6 \mathrm{H}-\mathrm{SiC}(115.0 \mathrm{eV}$, XES) were measured at 25, 300, 600 and $900{ }^{\circ} \mathrm{C}$ sample temperature. The single crystal was oriented such that the angle between the incoming $\mathrm{x}$-ray beam and the surface normal was 20 degrees for the XAS measurements to avoid self-absorption and 70 degrees for the XES and RIXS measurements to maximize count rate. The angle between the incoming $\mathrm{x}$-rays and the RIXS spectrometer was fixed at 90 degrees. The x-ray polarization was horizontal, e.g., parallel to the scattering 
plane. A typical XAS spectrum was measured in 20 minutes and XES and RIXS spectra were measured within 60 minutes with an average count rate of 120-200 counts per second.

In this study we use the term RIXS for excitation energies on the $\mathrm{Si} 2 \mathrm{p}$ resonance. For spectra with excitation energies in the continuum, that is when energies well above the $\mathrm{Si} 2 \mathrm{p}$ resonance are used, we refer to nonresonant $\mathrm{x}$-ray emission spectroscopy (XES). In short, XES and RIXS are two-photon processes. The incoming $\mathrm{x}$-ray photon excites a dipole transition, in our present study, a Si 2p core electron to an unoccupied level (either in or below the conduction band (RIXS) or in the continuum (for XES) depending on the excitation energy). When this created Si 2 p core hole is filled through a radiative decay from an occupied state, a photon is emitted. The energy and relative number of the emitted photons are measured with the spectrometer of our RIXS setup.

\subsection{Band-gap analysis from XAS and RIXS}

For analysis of the band gap with x-ray methods we have to take the following schematic figure (Figure 1) into account. This schematics show that an estimate of the 'real' CBM with XAS is disturbed by an exciton and by broadening in the Si $2 p$ core hole state. In our following analysis, the energy point of the half-way maximum of the first XAS band is used as the CBM estimate.
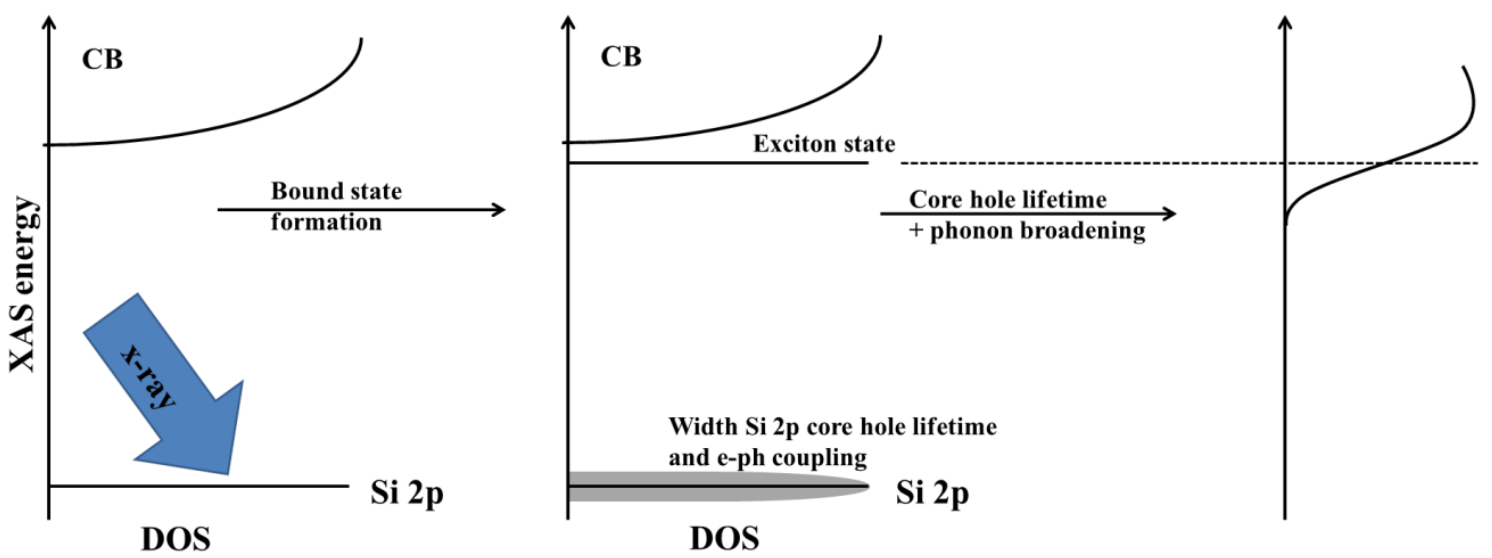

Figure 1. Schematic picture of the density of states (DOS) of the Si $2 p$ level and the conduction band on the left before absorption of an incident $x$-ray photon, in the middle after the creation of a bound exciton state and on the right after including the width of the Si $2 p$ core hole leading to the XAS spectrum.

This treatment of the XAS accounts for the existence of a core-excitonic state within the XAS edge [22] in contrast to the expected smooth onset of the conduction-band DOS (see CB DOS in Figure 1). This coreexcitonic state leads to an excitonic screening-energy contribution in the CBM which can be estimated in our band gap analysis as well. Note that Buczko et al. discussed the effect of the core hole exciton for the Si $2 \mathrm{p}$ spectrum of silicon [22]. They concluded that a calculation that uses an extra core charge (Z+1 approximation) or even a Bethe-Salpeter Equation (BSE) calculation, which includes both the core-hole and the excited electron in a neutral transition, is required for a simulation that fits onto experimental XAS. Thus, for a XAS transition in silicon and silicon compounds, inclusion of the excitonic properties in the analysis is important. This means that for Si 2p XAS, excitons are present and influence the energy assignment of the CBM as was found before for silicon [20]. In addition, we assume that the broadening of the XAS edge with increasing temperature is mainly a consequence of the (phonon) broadening of the Si 2 p core level as seen by temperature-dependent core level photoemission spectroscopy [23-25]. There are indications that e-ph coupling is larger for localized electronic states [26]. Since core levels are much more localized than valence states, this justifies our reasoning that the $\mathrm{Si}$ $2 p$ hole state is mainly broadened by phonons with increasing temperature.

For the XES spectra measured following an X-ray excitation (of $115.0 \mathrm{eV}$ ) into the continuum (and as well for RIXS spectra), the analysis is done more straight-forward: the zero-intensity crossing of a tangent set at halfway of the XES (RIXS) band maximum around $98 \mathrm{eV}$ is the estimated VBM. The spectral weight above this line is 
partially attributed to the limited resolution of the spectrometer and partially to a multi-electron response to the excitation. In the VBM analysis the XES spectra are used (instead of the RIXS spectra) to avoid any influence from the elastic X-ray scattering line. Overall, our quantitative analysis of the band gap reduction is similar to reference [20]. The VBM obtained from the XES is related to emission to both the $\mathrm{Si}_{3}$ and $\mathrm{Si} \mathrm{L}_{2}$ core level, while the CBM based on Si 2p XAS is strictly related to the $\mathrm{Si}_{3}$ hole. To account for this difference between CBM and VBM, the VBM is shifted with $-0.2 \mathrm{eV}$ (1/3 of the Si $2 p$ spin-orbit splitting, following the 2/3:1/3 ratio of $\mathrm{L}_{3}: \mathrm{L}_{2}$-edge) in order to have the VBM solely account for the emissions to the $\mathrm{Si}_{3}$ reference level. For the RIXS spectra, the energy difference between the elastic line $(101.35 \mathrm{eV})$ and the zero-intensity crossing of the tangent of the RIXS band around $98 \mathrm{eV}$ (as for the XES) is taken as another measure for the band gap.

\section{Results}

\subsection{Si $2 p$ XAS and XES results}

Figure 2 shows the (zoom-in of the) measured Si 2p XAS (at energies above $100 \mathrm{eV}$ ) and the Si 2p XES $(\mathrm{hv}=115 \mathrm{eV})$. The Si 2p XAS spectra are normalized to the bump maximum as visualized on the right hand side of Figure 1. All the Si 2p XES spectra are normalized to their area. The Si 2p XAS and XES spectra of $6 \mathrm{H}-\mathrm{SiC}$ measured at room temperature (black lines) agree reasonably well with the $6 \mathrm{H}-\mathrm{SiC}$ data published by Lüning et al. [27].

The Si 2p XAS spectra in Figure 2 measured at different temperatures show that the $\mathrm{Si}_{3}$ absorption edge position shifts to lower energy for measurements at higher temperature. For the XES (Figure 2, left side $\mathrm{hv}=115.0 \mathrm{eV}$ ), there is a shift of the band between 97-100 eV to higher energy with increasing temperature. Thus, the $\mathrm{Si} \mathrm{L}_{3}$ XAS edge shifts to lower energy and the 97-100 eV band in the Si $2 \mathrm{p}$ XES shifts to higher energy with increasing temperature. These observations imply that we can follow the decrease of the gap between the valence- and conduction-band with increasing temperature.

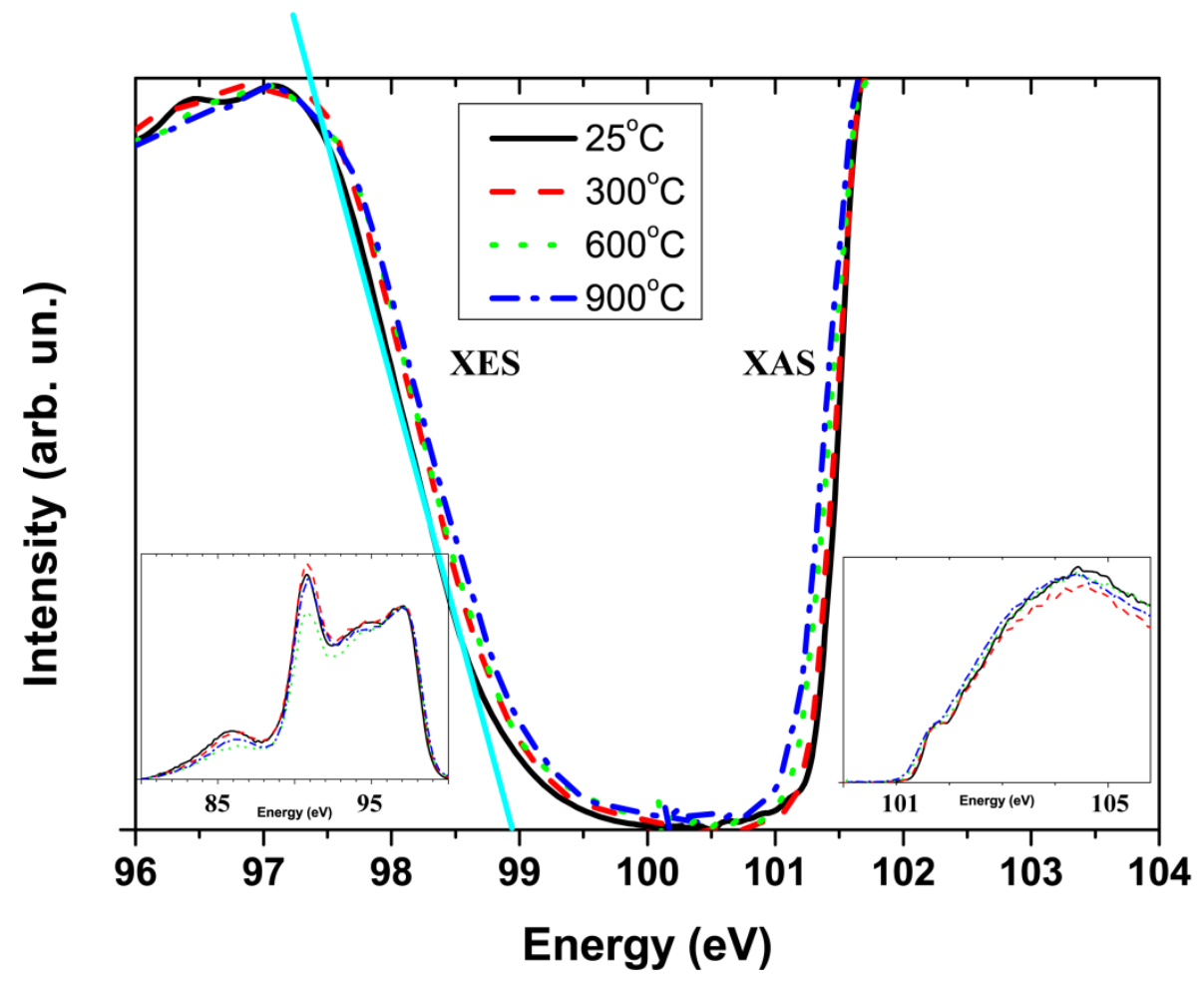

Figure 2. Experimental X-ray absorption (XAS) and X-ray emission (XES) spectra. Temperature-dependent zoom in of both Si 2p XAS and Si 2p XES (with incident $x$-ray energy hv=115.0 eV) of 6H-SiC. On the sides, there are insets of the complete Si 2p XES (left) and Si 2p XAS (right). The XES spectra are normalized to unit area. The determination procedure of the valence band maximum is displayed with a solid cyan line. 
As mentioned, for the XES the VBM analysis is performed using the zero-intensity crossing of a tangent set at halfway of the XES band maximum around $98 \mathrm{eV}$. As an example, a tangent for the XES measured at room temperature is shown in the top panel of Figure 2 with a cyan solid line.

The estimated VBM and CBM are shown in Figure 3A. It shows that this combination of XAS and XES method - in contrast to optical spectroscopy - is able to separate the valence-band and the conduction-band contributions to the temperature-dependent band-gap evolution. This is possible, since the Si $2 p$ orbital acts as an independent reference point in our case. Lüning et al. performed soft $\mathrm{x}$-ray absorption and emission spectroscopy on several $\mathrm{SiC}$ polytypes at room temperature [27] and they also analyzed the band gap (with both Si L- and C K-edge) with the difference between XAS and RIXS (that is with an x-ray excitation energy on the Si $2 p$ resonance). Lüning et al. used a linear extrapolation for both the XAS and RIXS edges to the baseline, which lead to a value of the band gap in the range of the optical band gap, however still leaving a $0.1 \mathrm{eV}$ difference with the optical band gap. By linear extrapolation on the XAS, their estimate for the CBM is roughly $0.3 \mathrm{eV}$ lower than ours would be. The consequence of taking the linear extrapolation of the XAS is that Lüning et al. do not take the core hole screening from the XAS process into account in their estimate, overall leading to band gaps that are closer to the optical band gap. As well they use the RIXS spectra (while we use the non-resonant XES), thus their VBM is estimated in the presence of the valence-exciton that partially screens the VBM. These differences make it difficult to directly compare the results of Lüning et al. for $6 \mathrm{H}-\mathrm{SiC}$ with our estimates.

\subsection{Band-gap results from XAS, XES and RIXS}

The estimated VBM and CBM as function of temperature both show linear behavior. Linear fits of the CBM with $\mathrm{Y}^{\mathrm{CBM}}=101.528(4)-1.334(5) \times 10^{-4} \mathrm{~T}$ (red dotted line) and $\mathrm{VBM}$ with $\mathrm{Y}^{\mathrm{VBM}}=99.05(4)+2.45(5) \times 10^{-4} \mathrm{~T}$ (black dotted line) are shown in Figure 3A. Based on these fitted lines it can be concluded that the band gap shrinking with temperature of $6 \mathrm{H}-\mathrm{SiC}$ is asymmetric, e.g., about $65 \%$ of the band gap shrinking comes from the slope of the valence band maximum, $+2.45 \times 10^{-4} \mathrm{eV} / \mathrm{K}$.

Asymmetric band gap closing was also obtained for silicon [20], where the absolute temperature-dependent slope of the VBM $\left(+2.93 \times 10^{-4} \mathrm{eV} / \mathrm{K}\right)$ was higher than the slope of the CBM $\left(-1.61 \times 10^{-4} \mathrm{eV} / \mathrm{K}\right)$. The observations for silicon gave experimental evidence for the combination of the theoretical treatments of Lautenschlager et al. [28] on one side and Cardona and Gapolan [13] on the other side. The data for $6 \mathrm{H}-\mathrm{SiC}$ show that the asymmetry in band gap shrinking is not a peculiarity of silicon, but may be more generally related to indirect band gap materials (or maybe even to all semiconductors and insulators). Since the VBM and CBM are at different points in k-space, they have a different symmetry and naturally, thermally induced phonons interact differently with those different states, resulting in different slopes of the CBM and VBM with temperature. At higher temperature, there are more phonons and therefore the atomic positions deviate more from their equilibrium crystalline sites. In turn, this leads to band edges becoming more fuzzy and broader. That is why the VBM increases, while the CBM decreases with temperature. 


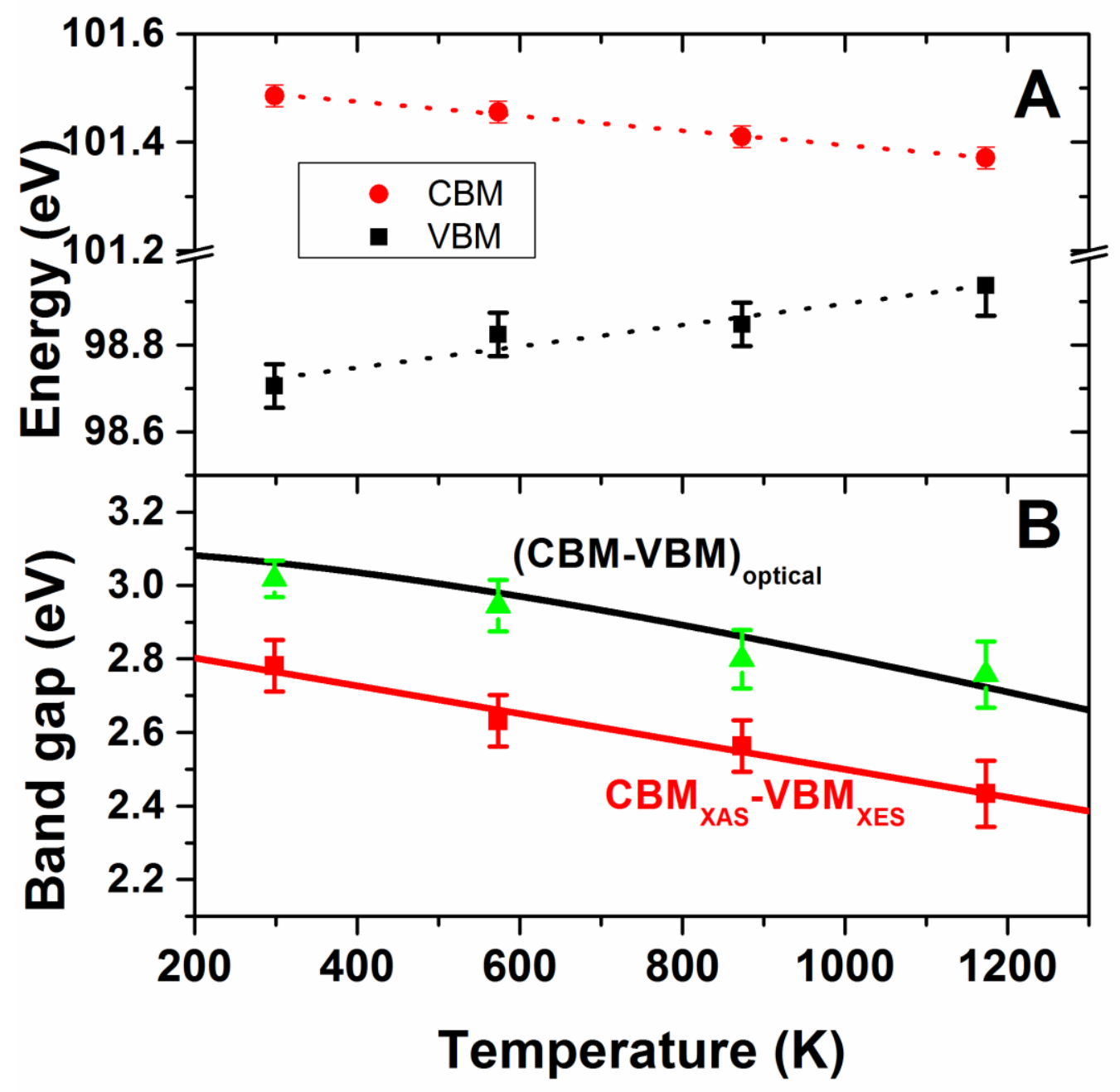

Figure 3. Asymmetric band gap closing in $6 \mathrm{H}-\mathrm{SiC}$. A) Valence band maximum (VBM) and conduction band minimum (CBM) derived from the XAS and XES as a function of temperature. B) From XAS and XES determined experimental $\mathrm{CBM}_{\mathrm{XAS}}-\mathrm{VBM}_{\mathrm{XES}}$ band gap (red squares) and fit (red line) as a function of temperature. The optical band gap with $\mathrm{E}_{\mathrm{g}}(0)=3.1 \mathrm{eV}[10,18]$ (black solid line) is shown for comparison as well as the estimated energy loss

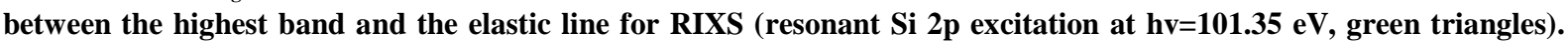
The RIXS spectra can be found in Ref. [26]. The temperature-independent off-set is due to the screening of the XAS core-excited final state.

The overall band gap decrease as a function of temperature is simply the absolute value of the difference of the obtained VBM and CBM slopes, e.g., $2.45(5) \times 10^{-4}+1.334(5) \times 10^{-4}=3.78(5) \times 10^{-4} \mathrm{eV} / \mathrm{K}$ in the studied temperature range. Applying a linear fit on the literature temperature relation of the $6 \mathrm{H}-\mathrm{SiC}$ band gap from Ref. [10] in the same temperature range provides a slope of $3.9 \times 10^{-4} \mathrm{eV} / \mathrm{K}$, confirming the validity of our method. In Figure 3B the optical band gap (CBM-VBM) $)_{\text {optical }}$ and our obtained $(\mathrm{CBM}-\mathrm{VBM})_{\mathrm{x} \text {-ray }}$ band gap obtained using $(\mathrm{CBM}-\mathrm{VBM})_{\mathrm{x}-\mathrm{ray}}=\mathrm{CBM}_{\mathrm{XAS}}-\mathrm{VBM}_{\mathrm{XES}}$ as described above as a function of temperature are shown for comparison. The temperature dependence of the band gap as measured with XAS and XES follows the trend of the optical band gap, but there is a temperature-independent offset between the optical band gap and the band gap estimated from the difference between XAS and XES. 


\section{Discussion}

\subsection{Core-excitonic screening energy}

As mentioned above, the CBM analysis based on XAS includes an effect of the Si $2 p$ core hole, thus the band gap size is influenced: a well-screened core-excitonic state appears at lower energy than the conduction-band DOS. In the XES, the final state contains a hole in the valence-band, which is a small perturbation as compared to the core-excited XAS final state. We assume that the electron-hole interaction in the optical case is much weaker. Therefore, the difference between the optical band gap and the band gap determined from the XAS and XES edge positions leads directly to the core excitonic screening energy, $\mathrm{E}_{\text {screen }}$ : $\mathrm{E}_{\text {screen }}=(\mathrm{CBM}-\mathrm{VBM})_{\text {optical }}-$ $\left(\mathrm{CBM}_{\mathrm{XAS}}-\mathrm{VBM}_{\mathrm{XES}}\right)$. The observed off-set is temperature-independent and $\mathrm{E}_{\text {screen }}$ is $0.35(3) \mathrm{eV}$ for $6 \mathrm{H}-\mathrm{SiC}$. This is about twice the screening energy obtained for silicon [20]. The core-excitonic screening energy of $6 \mathrm{H}-\mathrm{SiC}$ is about five times larger than the valence-exciton binding energy of $6 \mathrm{H}-\mathrm{SiC}$ in Table 1 proving the correctness of our assumption above, e.g., that a hole in the valence-band is a small perturbation compared to the core-excited XAS final state.

Additionally the energy difference between the elastic scattering $(101.35 \mathrm{eV})$ of the RIXS and the estimated valence-band maxima (same procedure as XES) of these RIXS spectra at different temperatures (green triangles) was shown in Figure 3B. These estimated energy differences for the RIXS correspond very well to the optical band gap including its temperature-evolution.

Figure 4 provides a scheme for the different XES, RIXS and optical processes and shows the physical origin for the difference between the VBM-CBM estimate for RIXS and the VBM estimate for XES (XES in Figure 4). Starting from a particular ground state (GS), it shows the non-resonant XES process in the left and the RIXS on the right side. The excitation process of XES on the left leads to a Si 2p core hole state (core hole). Further this process leads to a delocalized (possibly free) electron, which energy and work function are neglected in this scheme, but this means that these continuum excitations lead to core hole charged states. If a radiative decay occurs from such a core hole state, the only occupied states that can decay into the created $\mathrm{Si} 2 \mathrm{p}$ core hole are occupied valence states leaving behind valence hole states (valence hole). The schematic figure shows a box named valence hole to illustrate that these final states contain a bunch of possible valence holes due to the amount of electrons available in the valence band. This reflects the electronic DOS of the valence-band and leads to the observation of a band in the XES (and also in the RIXS) spectrum. Nevertheless, in this research paper we are mainly interested in the valence hole at lowest energy which corresponds to the highest available $\mathrm{x}$-ray emission energy (smallest difference between fixed $\mathrm{x}$-ray excitation energy and $\mathrm{x}$-ray emission energy) and thus to the bottom of the box in the schematic figure. 


\section{Core hole charged states}

\section{Neutral excitations}

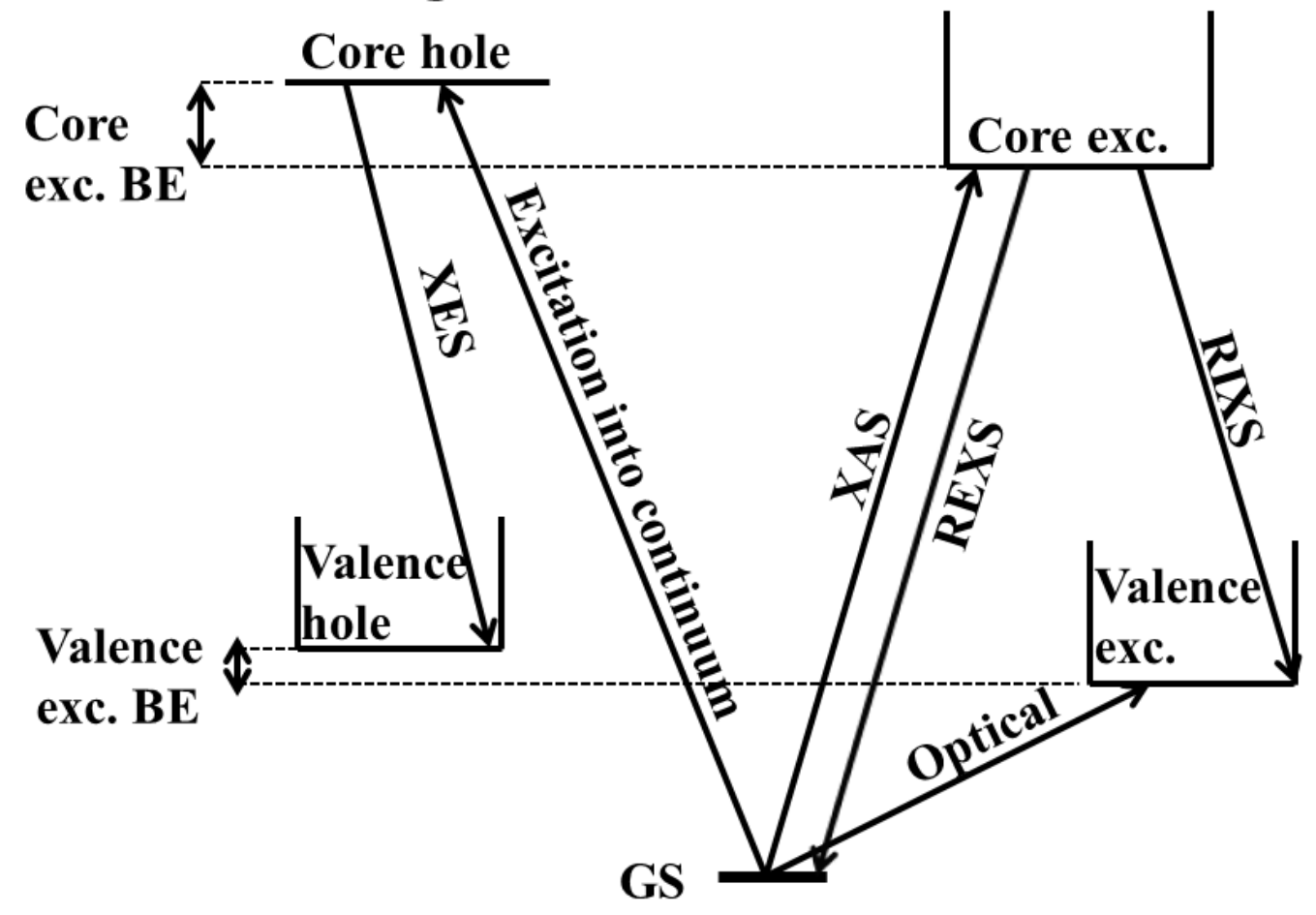

Figure 4. Schematic total energy diagram of XAS, an electronic excitation into continuum and of an optical transition.

On the right side of Figure 4 the process of RIXS is shown schematically. There the x-ray excitation (XAS) leads to an electron in a localized state just below the conduction band that is still closely linked to the Si $2 p$ core hole. This is a core-hole exciton and we refer to a core excitonic binding energy (core exc. BE) as compared to the core hole state on the left. From this core excited state, radiative decay can occur in two ways. (1) Either the excited electron decays back in to the $\mathrm{Si} 2 \mathrm{p}$ core, which is elastic x-ray scattering (Resonant Elastic X-ray Scattering, REXS) and appears in a RIXS spectrum as the elastic line or (2) electrons from the valence band decay back into the created Si 2p core hole leaving behind the electron in the conduction band. Therefore the second process leaves a combination of a valence hole and a conduction electron, or in other words a valence exciton. Again these valence exciton final states can be of different energy due to decay of any valence electron with different energy. Therefore a valence exc. box is shown in Figure 4. This box of final states can also be reached with optical or thermal band-gap studies. Thus with this schematic Figure 4 it is clear that RIXS has a similar final state as the optical excitation: for both the optical excitation and RIXS, the final state is an excited electron in a so-called valence excited or valence excitonic state. That is the reason why the green triangles in Figure 3 agree so well with the temperature evolution of the optical band gap.

Nevertheless, one has to be careful to generalize the direct relationship of the optical process with RIXS, since RIXS is a two-photon process with two dipole transitions, while the optical band gap contains only one dipole transition. Thus, in case the VBM to CBM transition is not dipole-allowed, RIXS could still obtain the lowest band gap energy, while the optical transition of the lowest band gap would only be allowed if it is accompanied by e-ph coupling.

Another difference between measuring band gaps with optical or x-ray methods is the penetration depth. Although the penetration depth of optical and x-ray photons differ by an order of magnitude, both the optical and $\mathrm{X}$-ray emission methods are bulk-sensitive. Note that when X-ray valence band photoemission instead of XES or RIXS would be used as a measure for the VBM, it could create a difference between the optical band gap and the band gap obtained with X-ray methods, since X-ray photoemission is a surface-sensitive technique. Therefore Xray valence band photoemission would give a surface-VBM. 
Another point of attention is the following: since the RIXS process is related to a localized $\mathrm{Si} 2 \mathrm{p}$ core hole, RIXS leads to a local probe of the band gap and related excitons, while the optical band gap may contain both localized and delocalized excitons. This difference is also reflected in the time scales of the optical and x-ray absorption, which may suppress certain transitions in the case of optical studies. That could mean that optical band gap studies would lead to the determination of larger band gaps (but only if optical excitation transitions to the lowest conduction band states are suppressed) than RIXS.

Figure 4 provides that the valence exciton binding energy and the core exciton binding energy are different which is related to the difference in the screening of the nucleus by the electrons: the atomic shielding is stronger for the core electron than for the valence electron. As a consequence, a hole instead of an electron leads to a lower shielding, thus attraction of the electrons to the nucleus increases for a core hole compared to a valence hole [29]. Therefore, the core-exciton binding energy is bigger than the valence-exciton binding energy. Nevertheless, it should be in the same order of magnitude. Note that the exciton binding energies are exaggerated on the energy scale of Figure 4 compared to the $x$-ray excitation energies only to illustrate the differences between RIXS and non-resonant XES.

\subsection{Comparison silicon and $6 \mathrm{H}-\mathrm{SiC}$}

Comparing the results of $6 \mathrm{H}-\mathrm{SiC}$ to silicon, we have to keep in mind that the average mass of an atom in $\mathrm{SiC}$ is 20.05 (with silicon and carbon atoms in a 1:1 ratio) and 28.09 in silicon. Due to the lighter weight of the surrounding carbon atoms, the vibrational adaptation to changes in $6 \mathrm{H}-\mathrm{SiC}$ such as to the creation of a core hole, can lead to more extreme lowering of the energy of the final state with its excitonic screening energy, which might be related to the differences observed between the silicon and $6 \mathrm{H}-\mathrm{SiC}$ core-excitonic screening energies in Table 2. For both the $6 \mathrm{H}-\mathrm{SiC}$ and silicon the asymmetric band closing was about $65 \%$ due to the VBM shift with temperature and while both these materials have their VBM on $\Gamma$ in $\mathrm{k}$-space, the point of the k-space, where the $\mathrm{CBM}$ is found is different for $6 \mathrm{H}-\mathrm{SiC}(\mathrm{M} / \mathrm{L})$ and silicon $(\mathrm{X})$ (see Table 2) which could be another reason for differences in the core-excitonic screening energies between $6 \mathrm{H}-\mathrm{SiC}$ and silicon. It is noted that differences between the local valence DOS for $6 \mathrm{H}-\mathrm{SiC}$ are also expected to influence the core-hole structure and therefore the core-excitonic screening energy.

Table 2. Several band gap parameters for $6 \mathrm{H}-\mathrm{SiC}$ and silicon. If known, the standard deviation is presented in between parentheses.

\begin{tabular}{|l|c|c|}
\hline & 6H-SiC & Silicon \\
\hline Core-excitonic screening energy $(\mathrm{eV})$ & $0.35(0.03)$ & $0.17 *(0.02)$ \\
\hline Band gap (indirect) energy $(\mathrm{eV})$ & $3.1[10]$ & $1.12[10]$ \\
\hline Si 2p excitation to K-point & $\mathrm{M} / \mathrm{L}[10]$ & (close to) $\mathrm{X}[10]$ \\
\hline Band gap temperature dependence $(\mathrm{eV})$ & $\mathrm{E}_{\mathrm{g}}(0)-6.4 \times 10^{-4} \mathrm{~T}^{2} /(\mathrm{T}+1200)[10]$ & $\mathrm{E}_{\mathrm{g}}(0)-4.73 \times 10^{-4} \mathrm{~T}^{2} /(\mathrm{T}+636)[10]$ \\
\hline
\end{tabular}

*The estimated core-excitonic screening energy for silicon was estimated in Refs. [20,30] to be 0.37 eV. Here we have made a similar adjustment to relate the VBM solely to the $\mathrm{Si}_{3}$-hole for silicon as was done for $6 \mathrm{H}-\mathrm{SiC}$ as mentioned above in the experimental section.

The combination of XAS and XES is an alternative for measuring the band gap including the core-excitonic binding energy that shifts the assignment of the CBM with XAS. Although optical band gap studies are easier to perform, the XAS plus XES alternative can provide valuable information on the asymmetry of the band gap closing and one can obtain both an optical-like band gap using RIXS and the separate assignment of VBM and CBM with the combination of the XAS and non-resonant XES techniques. Information on the asymmetric band gap closing may be valuable for the use of either $\mathrm{n}$ - or $\mathrm{p}$-doped (or both $\mathrm{n}, \mathrm{p}$-doped) semiconductors and Schottky barriers occurring at the interface between different semiconductors for applications that need excellent performance at different temperatures. 


\section{Summary and conclusions}

Summarized, the band gap of $6 \mathrm{H}-\mathrm{SiC}$ was estimated with two different methods: (1) using XAS for the CBM assignment and XES for the VBM assignment, allowing the separate observation of CBM and VBM and as a consequence asymmetric closing and core exciton binding energies and (2) with the on-set of the energy loss of the first band below the elastic scattering using RIXS. This energy loss is a direct measure for the band gap with a similar final state as the optical excitation and relates very well to the temperature-dependence of the band gap found with optical studies. However, it is emphasized that the present method of determining the band gap is based effectively on non-dipolar (monopole or quadrupole) excitation (due to the two dipole transitions of RIXS) in contrast to the direct optical method. This becomes an advantage when indirect band gap materials are the study object.

\section{Acknowledgements}

We thank HZB for the allocation of synchrotron radiation beamtime.

\section{References}

1. C. Kittel, Introduction to solid state physics. reprint 2005.

2. K. Koga and T. Yamaguchi, Progress in Crystal Growth and Characterization of Materials, 1991. 23: p. $127-151$.

3. J. Edmond, H. Kong, A. Suvorov, D. Waltz and C. Carter Jr., Physica Status Solidi a-Applied Research, 1997. 162(1): p. 481-491.

4. M. Syväjärvi, J. Müller, J.W. Sun, V. Grivickas, Y. Ou, V. Jokubavicius, P. Hens, M. Kaisr, K. Ariyawong, K. Gulbinas, P. Hens, R. Liljedahl, M.K. Linnarsson, S. Kamiyama, P. Wellmann, E. Spiecker and H. Ou, Physica Scripta, 2012. T148: 014002.

5. S. Dakshinamurthy, N.R. Quick, and A. Kar, Journal of Physics D-Applied Physics, 2007. 40(2): p. 353-360.

6. F. Nava, G. Bertuccio, A. Cavallini and E. Vittoni, Measurement Science \& Technology, 2008. 19(10): 102001.

7. F.H. Ruddy, A.R. Dulloo, J.G. Seidel, M.K. Das, R. Sei-Hyung and A.K. Agarwal, Nuclear Science, IEEE Transactions on, 2006. 53(3): p. 1666-1670.

8. N. Bernstein, H.J. Gotsis, D.A. Papaconstantopoulos and M.J. Mehl, Physical Review B, 2005. 71(7): 075203.

9. J. Noffsinger, E. Kioupakis, C.G. Van de Walle, S.G. Louie and M.L. Cohen, Physical Review Letters, 2012. 108(16): 167402.

10. V. Siklitsky and A. Tolmatchev. Electronic archive New Semiconductor Materials. Characteristics and Properties, http://www.ioffe.ru/SVA/NSM/introduction.html. [visited: April 2013].

11. R.G. Humphreys, D. Bimberg, and W.J. Choyke, Solid State Communications, 1981. 39(1): p. 163-167.

12. M. Cardona and M.L.W. Thewalt, Reviews of Modern Physics, 2005. 77(4): p. 1173-1224.

13. M. Cardona and S. Gopalan, Temperature Dependence of the Band Structure of Semiconductors: Electron-Phonon Interaction, in Progress in Electron Properties of Solids. 1989, Springer Netherlands. p. 51-64.

14. R. Pässler, physica status solidi (b), 2003. 236(3): p. 710-728.

15. J. Bhosale, A.K. Ramdas, A. Burger, A. Munoz, A.H. Romero, M. Cardona, R. Lauck and R.K. Kremer, Physical Review B, 2012. 86(19): p. 195208.

16. D. Olguin, M. Cardona, and A. Cantarero, Solid State Communications, 2002. 122(11): p. 575-589.

17. Y.P. Varshni, Physica, 1967. 34(1): p. 149-154.

18. Y. Goldberg, M.E. Levinshtein, and S.L. Rumyantsev, eds. Properties of Advanced SemiconductorMaterials GaN, AlN, SiC, BN, SiC, SiGe. Properties of Advanced SemiconductorMaterials GaN, AlN, SiC, BN, SiC, SiGe ed. M.E. Levinshtein, S.L. Rumyantsev, and S. M.S. 2001, John Wiley \& Sons, Inc.,: New York. 93-148.

19. G.L. Harris, ed. Properties of Silicon Carbide. 1995, INSPEC: London, UK.

20. M. Beye, F. Hennies, M. Deppe, E. Suljoti, M. Nagasono, W. Wurth and A. Föhlisch, New Journal of Physics, 2010. 12(043011): 043011.

21. K. Kunnus, et al., Review of Scientific Instruments, 2012. 83(12): p. 8.

22. R. Buczko, G. Duscher, S.J. Pennycook and S.T. Pantelides, Physical Review Letters, 2000. 85(10): p. 2168-2171.

23. C. Grupp and A. Taleb-Ibrahimi, Journal of Electron Spectroscopy and Related Phenomena, 1999. 101103(0): p. 309-313. 
24. P. Unsworth, J.E. Evans, P. Weightman, A. Takahashi, J.A.D. Matthew, Q.C. Herd, Physical Review B, 1996. 54(1): p. 286-290.

25. K. Hricovini, R. Günther, P. Thiry, A. Taleb-Ibrahimi, G. Indlekofer, J.E. Bonnet, P. Dumas, Y. Petroff, X. Blase, X. Zhu, S.G. Louie, Y.J. Chabal and P.A. Thiry, Physical Review Letters, 1993. 70(13): p. 1992-1995.

26. P.S. Miedema, M. Beye, R. Könnecke, G. Schiwietz and A. Föhlisch, New J Phys, 2014. Under review.

27. J. Lüning, S. Eisebitt, J.-E. Rubensson, C. Ellmers and W. Eberhardt, Physical Review B, 1999. 59(16): p. 10573-10582.

28. P. Lautenschlager, P.B. Allen, and M. Cardona, Physical Review B, 1985. 31(4): p. 2163-2171.

29. J.C. Slater, Physical Review, 1930. 36(1): p. 57-64.

30. M. Beye and A. Fohlisch, Journal of Electron Spectroscopy and Related Phenomena, 2011. 184(3-6): p. 313-317. 